or Relative Risks). Another relevant problem is the presence of various confounding factors, e.g. occupational exposure to physical or chemical carcinogens possibly occurring in several workplaces, and others, potentially affecting the overall results. Conclusions The completion of studies collection, and data extraction and quality assessment of the papers including Risk of Bias analysis according to the protocol registered in PROSPERO, are currently ongoing.

\section{P-230 THE MENTAL HEALTH OF CHILEAN TEACHERS IN TIMES OF FORCED TELEWORK: HOW MANY, WHO AND WHY ARE THEY IN WORSE HEALTH?}

${ }^{1}$ Claudia Palma-Vasquez, Diego Carrasco, Julio C Hernando-Rodriguez. ${ }^{1}$ Pompeu Fabra University, Spain

\subsection{6/OEM-2021-EPI.242}

Introduction The impact of the COVID-19 pandemic on education resulted in school closures and the forced implementation of virtual teaching and teleworking. This situation together with the diversity of social and economic contexts in schools, has emphasized inequality in access to quality education and increased stress and anxiety among teachers.

Objectives This study aims to explore the mental health of teachers forced to telework because of COVID-19 and analyze its relationship with sociodemographic, teacher-related and working conditions.

Methods The sample was 278 Chilean classroom teachers who teleworked more than 50\% during the 2020 academic year. The dependent variable was mental health measured through the General Health Questionnaire (GHQ-12). The independent variables were sociodemographic, teacher-related and work conditions. The internal structure of the mental health construct was evaluated using the Rasch model. Crude (cOR) and adjusted odds ratios (aOR) were estimated using logistic regression models. The analyzes were stratified by years of teaching experience and sex.

Results A high prevalence of poor mental health was found in teachers $(58 \%)$. Working in a private-subsidized school (aOR $=2.89 ; 95 \% \mathrm{CI}: 1.16-7.22)$, working two or more unpaid overtime hours $(\mathrm{aOR}=2.25 ; 95 \% \mathrm{CI}: 1.11-4.59)$ and having sickness absence $(\mathrm{aOR}=3.82$; 95\% CI: 1.53 - 9.58) were associated with poor mental health. Working 35 hours or more weekly among less experienced teachers (6-10 years: $\mathrm{aOR}=0.07 ; 95 \% \mathrm{CI}: 0.01-0.51)$ and being a tutor teacher among women $(\mathrm{aOR}=0.48 ; 95 \% \mathrm{CI}: 0.23-1.0)$ had a protective effect on mental health.

Conclusion This study contributes to the recognition of a high prevalence of poor mental health among Chilean teachers and its associated contextual and labour factors. Need for actions to improve the working conditions of teachers who telework are guaranteed to improve their mental health.

\section{P-235 A DESCRIPTIVE ANALYSIS OF WORKSITE WELLNESS PROGRAM PARTICIPANTS IN A US DEPARTMENT OF ENERGY NATIONAL LABORATORY, 2013 TO 2019}

'Sara Howard, Ashley Golden. 'Oak Ridge Associated Universities (ORAU), United States
Introduction Since most working Americans spend one third of their day at a worksite, worksite wellness programs (WWP) provide an avenue for promotion of healthy lifestyles and may reduce health risks.

Objective The purpose of this study is to describe the health trends in the WWP and identify differences between WWP participants and non-WWP workers.

Methods Health data collected from WWP participants at a US Department of Energy National Laboratory was compared to other site personnel who visited Occupational Health services during program years 2013 to 2019. Data were analyzed continuously and categorically, and analyses were conducted in SAS and R.

Results The number of participants in the WWP varied each year (range: 1,463-2,556), with participation slightly declining in later years. Overall, the percentage of those with normal blood pressure declined from $44.7 \%$ in 2013 to $29.9 \%$ in 2019, while those with a normal total cholesterol increased from 58\% in 2013 to $67.2 \%$ in 2019. Both males and females in WWP saw changes in waist circumference, blood pressure, and pulse across the program years. Trends in blood pressure showed a decline in normal readings, but both waist circumference and pulse increased the percentage of normal readings. When comparing WWP participants to non-participants, WWP generally had better health values than non-participants in heart rate and blood pressure. In most years, BMI remained the similar between the two groups; however, WWP participants had significantly lower BMIs in 2013 and 2015.

Conclusion The results of the study show that biometric values change over time for WWP participants. Overall, the WWP participants had better biometric values than non-participants. However, more research is needed to determine if this difference is the result of the WWP, or if the volunteerism of WWP participants represents a behavioral difference that may influence their willingness to improve their health.

\section{P-239 DETERMINANTS OF MUSCULOSKELETAL DISORDERS OF THE UPPER LIMBS AMONG MILITARY PERSONNEL IN TUNISIA.}

'Ons Grissa, Ines Rassas, Taoufik Slimene, Marouen Hayouni, Charfeddine Amri, Adel Amri, Adnene Hanchi, Neila Chaari, Mohamed Akrout, Irtyah Merchaoui. ' Faculty of Medicine of Monastir, University of Monastir, Tunisia

\subsection{6/OEM-2021-EPI.244}

Introduction Musculoskeletal disorders (MSDs) are the first medical reason for work stoppage among military personnel and are one of the main reasons for dismissal from army service. Prevalence of MSDs, in particular those of the upper limbs (UL-MSDs), among military officers, is insufficiently known, as well as their risk factors, given the complexity of the military service.

Objective To assess the prevalence and the determinants of UL-MSDs in Tunisian army officers.

Methods This is a cross-sectional study among army officers, based on a questionnaire with several items: socio-demographic, occupational characteristics, and psycho-social constraints at work through the Karasek standardized questionnaire. The Nordic questionnaire was adapted to assess musculoskeletal health. 